\title{
Long Noncoding RNA EZR-AS1 Regulates the Proliferation, Migration, and Apoptosis of Human Venous Endothelial Cells via SMYD3
}

\author{
Ganhua You $\mathbb{D}$, ${ }^{1,2}$ Xiangshu Long $\mathbb{D}^{3},{ }^{3}$ Fang Song $\left(\mathbb{D},{ }^{3}\right.$ Jing Huang $\left(\mathbb{D},{ }^{3}\right.$ Maobo Tian $\mathbb{D}{ }^{3}$ \\ Yan Xiao $₫{ }^{3}$ Shiyan Deng $\left({ }^{3},{ }^{3}\right.$ and Qiang $\mathrm{Wu} \mathbb{1}^{3}$ \\ ${ }^{1}$ Guizhou University School of Medicine, No. 2708 South Section of Huaxi Avenue, Guiyang 550025, China \\ ${ }^{2}$ Guizhou Institute for Food and Drug Control, No. 142 Shibei Road, Guiyang 550004, China \\ ${ }^{3}$ Department of Cardiology, Guizhou Provincial People's Hospital, People's Hospital of Guizhou University, No. 83 Zhongshan \\ East Road, Guiyang 550002, China
}

Correspondence should be addressed to Qiang Wu; wqgz0851@126.com

Received 30 December 2019; Accepted 25 February 2020; Published 27 May 2020

Academic Editor: Bo Zuo

Copyright ( $) 2020$ Ganhua You et al. This is an open access article distributed under the Creative Commons Attribution License, which permits unrestricted use, distribution, and reproduction in any medium, provided the original work is properly cited.

\begin{abstract}
Numerous studies have shown that long noncoding RNAs (lncRNAs) play essential roles in the development and progression of human cardiovascular diseases. However, whether lncRNA ezrin antisense RNA 1 (EZR-AS1) is associated with the progression of coronary heart disease (CHD) remains unclear. Accordingly, the aim of the present study was to evaluate the role of lncRNA EZR-AS1 in patients with CHD and in human venous endothelial cells (HUVECs). The findings revealed that lncRNA EZR-AS1 was highly expressed in the peripheral blood of patients with CHD. In vitro experiments showed that the overexpression of EZR-AS1 could enhance proliferation, migration, and apoptosis by upregulating the expression of EZR in HUVECs; downregulation of lncRNA EZR-AS1 resulted in the opposite effect. IncRNA EZR-AS1 was also found to regulate SET and MYND domain-containing protein 3 (SMYD3), a histone H3 lysine 4-specific methyltransferase, which subsequently mediated EZR transcription. Collectively, these results demonstrate that lncRNA EZR-AS1 plays an important role in HUVECs function via SMYD3 signaling.
\end{abstract}

\section{Introduction}

As a result of economic development and an aging population, the risk of developing cardiovascular disease has gradually increased $[1,2]$. Coronary heart disease (CHD) is a common, high rate of mortality disease with global influence $[3,4]$, and it is therefore necessary to optimize treatment and prevention strategies.

Long noncoding RNAs (lncRNAs) are a class of RNA molecules $>200$ nucleotides in length, which do not encode proteins $[5,6]$. IncRNAs regulate gene expression through epigenetic, transcriptional, and post-transcriptional mechanisms. Recent studies have shown that IncRNAs are also involved in the development and progression of various cardiovascular diseases [7-11], including heart failure, myocar- dial hypertrophy, heart metabolic disease, myocardial infarction, and atherosclerosis (AS).

Ezrin (EZR) is a member of the ezrin-radixin-moesin (ERM) family of cytoskeletal proteins, which connects the actin cytoskeleton to the plasma membrane. EZR plays a vital role in numerous processes associated with normal cell growth, including adhesion, cell polarity, and migration $[12,13]$. SET and MYND domain-containing protein 3 (SMYD3) is a member of the SET and MYND domain (SMYD) family. Studies have shown that SMYD3 interacts with the specific region of trimethylation of histone $\mathrm{H} 3$ lysine 4- (H3K4Me3-) modified histone tails, which contributes its recruitment to the key promoter regions of downstream genes and activates the gene expression [14, 15]. Further studies indicate that SMYD3 could be recruited to SMYD3 


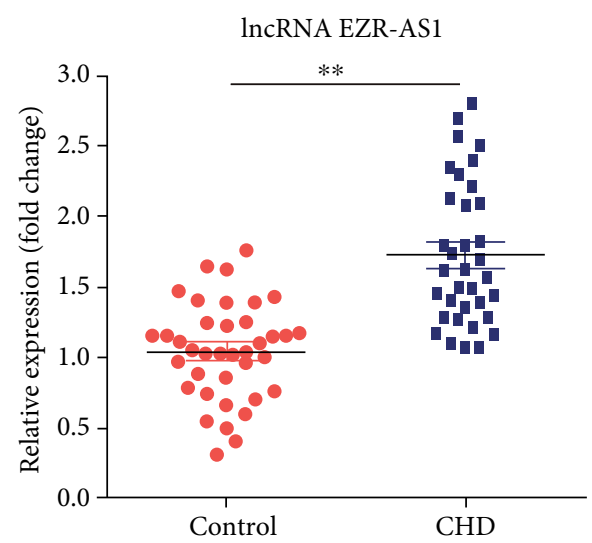

(a)

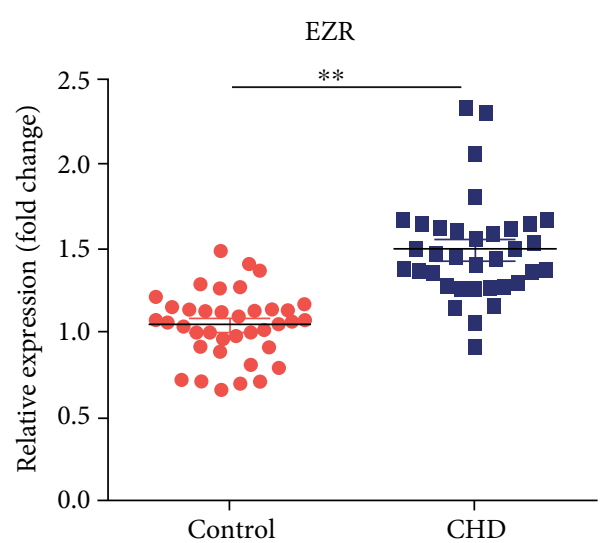

(b)

FIgURE 1: Expression of lncRNA EZR-AS1 and EZR in peripheral blood. Reverse transcription-quantitative PCR was used to detect the expression levels of (a) lncRNA EZR-AS1 and (b) EZR in the peripheral blood of control subjects and patients with CHD. All results were expressed as mean $\pm \mathrm{SD}$. ${ }^{* *} P<0.01$ vs. controls.

binding sites, which present in the downstream of the EZR promoter, causing local accumulation of SMYD3 and accompanied $\mathrm{H} 3 \mathrm{~K} 4 \mathrm{Me} 3$ at the EZR gene [16]. IncRNA EZR antisense RNA 1 (EZR-AS1) is $362 \mathrm{bp}$ in length and is located on chromosome 6q25.3. The expression of EZR-AS1 may promote cell migration and mediate cancer cell differentiation [17]. Despite these discoveries, the role of lncRNA $E Z R-A S 1$ in noncancerous pathologies, particularly cardiovascular diseases, remains unclear.

Therefore, the aim of the present study was to determine the function of EZR-AS1 in CHD, by assessing the proliferation, migration, and apoptotic rates of human venous endothelial cells (HUVECs) following the knockdown and overexpression of lncRNA EZR-AS1.

\section{Materials and Methods}

2.1. Clinical Blood Samples. In the present study, 35 patients (24 men and 11 women; 50-75 years of age) were recruited, who had been angiographically diagnosed with CHD at the Guizhou Provincial People's Hospital (Guiyang, Guizhou, China) between June 2018 and August 2018. In addition, 38 individuals without CHD (22 men and 16 women; 50-75 years of age) were enrolled as controls. The inclusion criteria included patients with stable angina pectoris exhibiting $\geq 1$ major coronary artery with $>80 \%$ stenosis. The exclusion criteria included patients with (i) unstable angina or myocardial infarction; (ii) CHD complicated with other organic heart diseases; and (iii) CHD combined with severe liver disease, kidney diseases, familial hypercholesterolemia, malignant tumors, or inflammatory diseases. All blood samples were immediately stored at $4^{\circ} \mathrm{C}$, and RNA was extracted on the same day. The study protocol was approved by the Human Ethics Committee Review Board of the Guizhou Provincial People's Hospital, and oral informed consent was obtained from each patient (Ethics approval No. (2019)068).

2.2. Cell Culture and Transfection. HUVECs were purchased from the Xiangya Cell Bank of Central South University. The cells were cultured in Roswell Park Memorial Institute
(RPMI) 1640 medium (HyClone; GE Healthcare Life Sciences) supplemented with $10 \%$ fetal bovine serum (Biological Industries) and $1 \%$ penicillin/streptomycin (Beijing Solarbio Science \& Technology Co., Ltd.), at $37^{\circ} \mathrm{C}\left(5 \% \mathrm{CO}_{2}\right)$. Cells in the logarithmic phase were harvested for further experimentation. The HUVECs were transiently transfected with negative control small interfering (si) RNA (si-control) or si-EZR$A S 1$; the pcDNA-control, pcDNA-EZR-AS1, pcDNA-EZR, or pcDNA-SMYD3; si-EZR-AS1+pcDNA-EZR; or si-EZR$A S 1+$ pcDNA-SMYD3 as appropriate. Transfection was carried out with $50 \mathrm{nM}$ siRNA or $1.6 \mu \mathrm{g} / \mathrm{ml}$ pcDNA using Lipofectamine 2000 (Invitrogen; Thermo Fisher Scientific, Inc.) according to the manufacturer's instructions. When simultaneously transfecting siRNA and pcDNA, $50 \mathrm{nM}$ siRNA and $1.6 \mu \mathrm{g} / \mathrm{ml}$ pcDNA were also used. The sequences of the siRNAs are as follows: si-EZR-AS1, $5^{\prime}$-UAUUUUCCAAAUC UUUUCCTT-3' ; si-SMYD3, $5^{\prime}$-UCACAGCUGUGACCCC AACTT-3'; and si-EZR, $5^{\prime}$-GCUCAAAGAUAAUGCUA UGTT-3'.

2.3. Cell Counting Kit-8 (CCK-8) Assay. HUVECs were seeded into 96-well culture plates at a density of $3 \times 10^{3}$ cells/well. The following day, the HUVECs were transfected with the corresponding siRNAs or overexpression pcDNA for 24,48 , and $72 \mathrm{~h}$. Cell viability was assessed using the CCK-8 assay (Dojindo Molecular Technologies, Inc.) according to the manufacturer's protocol; $10 \mu \mathrm{l} \mathrm{CCK-8} \mathrm{solution} \mathrm{was}$ added to each well, and the plates were incubated for $2 \mathrm{~h}$. The absorbance was then measured at $450 \mathrm{~nm}$ using a plate reader.

2.4. Wound Healing Assay. HUVECs were plated in 6-well cell culture plates, and $48 \mathrm{~h}$ after transfection, the cell monolayers were scratched with a $200 \mu \mathrm{l}$ plastic pipette tip. After washing twice with phosphate-buffered saline (PBS), the cells were incubated for $24 \mathrm{~h}$ in low-serum medium. The wound closure distance was then determined using an inverted microscope (Olympus Corporation) at $\times 100$ magnification, and the area of closure between the 0 and $24 \mathrm{~h}$ time points was calculated. 


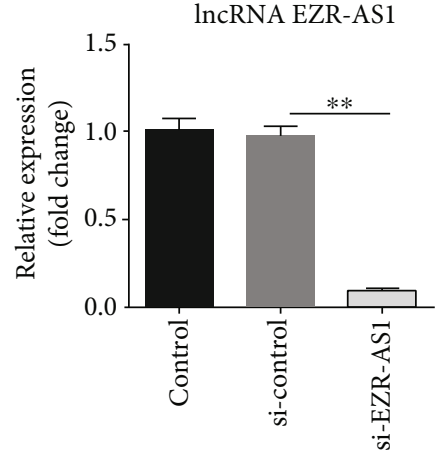

(a)

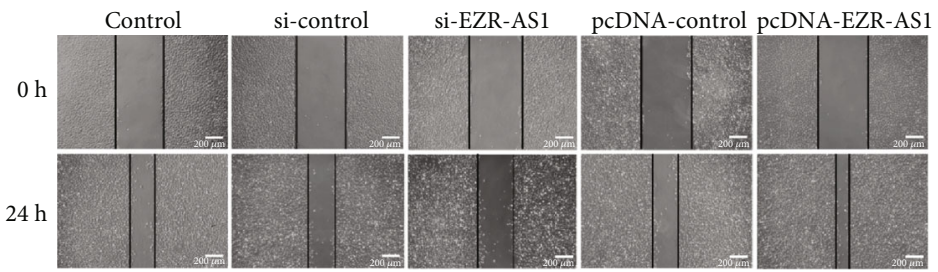

(c)

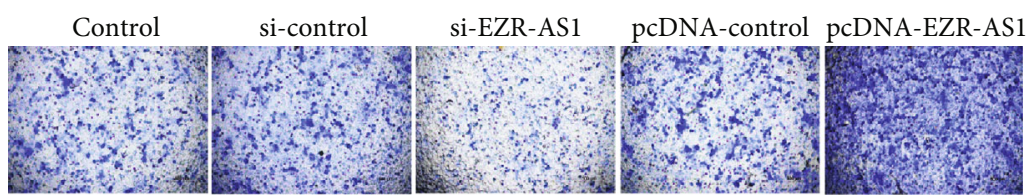

a)

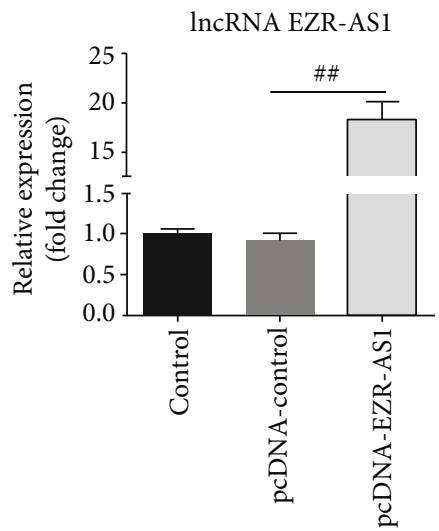

(d)

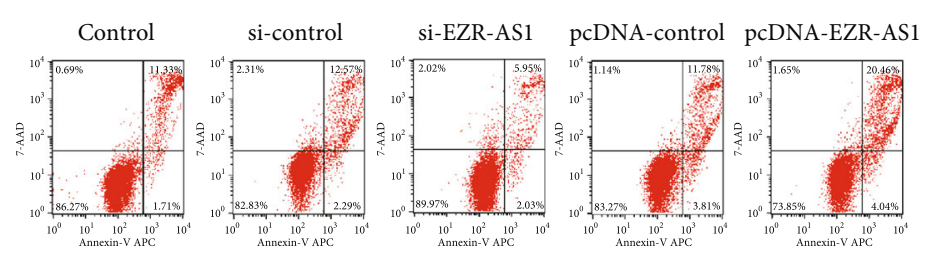

(e)

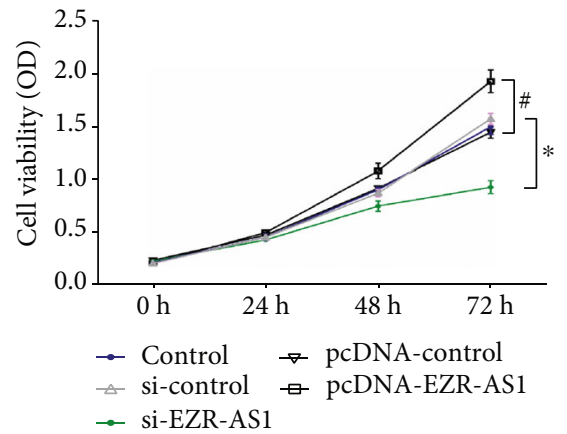

(b)
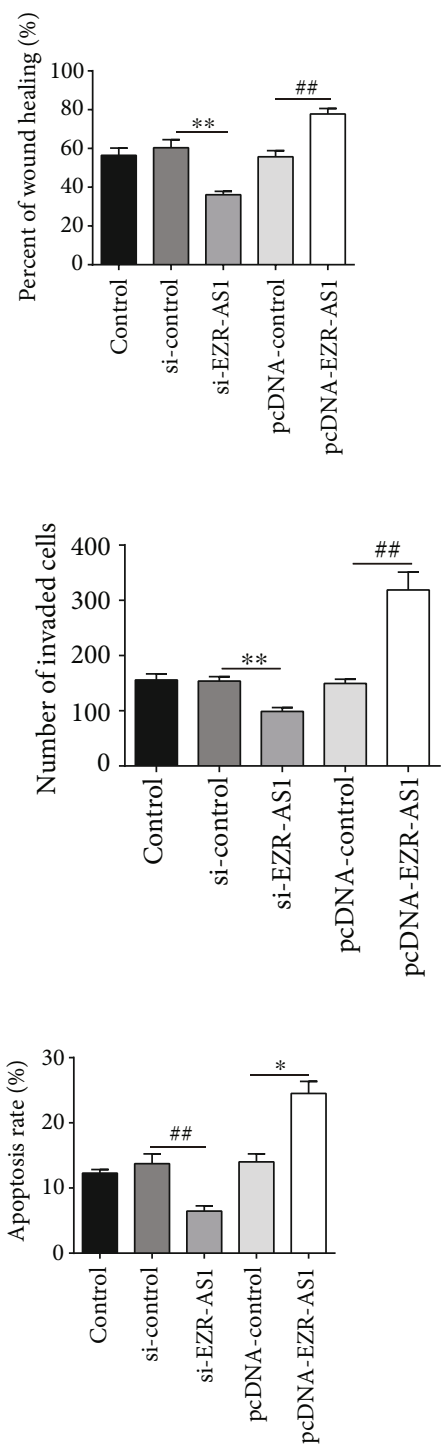

FIGURE 2: Effects of lncRNA EZR-AS1 on human venous endothelial cell proliferation, migration, and apoptosis. Cells were transfected with (a) lncRNA si-EZR-AS1 (knockdown) or si-control and with pcDNA-EZR-AS1 (overexpression) or pcDNA-control. Untreated cells were used as a control. Posttransfection with si-EZR-AS1 or pcDNA-EZR-AS1, (b) viability, (c, d) migratory ability, and (e) apoptotic rates of the cells were detected by CCK-8 assay, wound healing assay, Transwell assay, and flow cytometry, respectively. Wound healing was quantified, and the number of migratory cells was counted. Magnification, $\times 100$ (migration). All results were expressed as mean \pm SD. ${ }^{*} P<0.05$ and ${ }^{* *} P<0.01$ vs. si-control; ${ }^{\#} P<0.05$ and ${ }^{\# \#} P<0.01$ vs. pcDNA-control. 


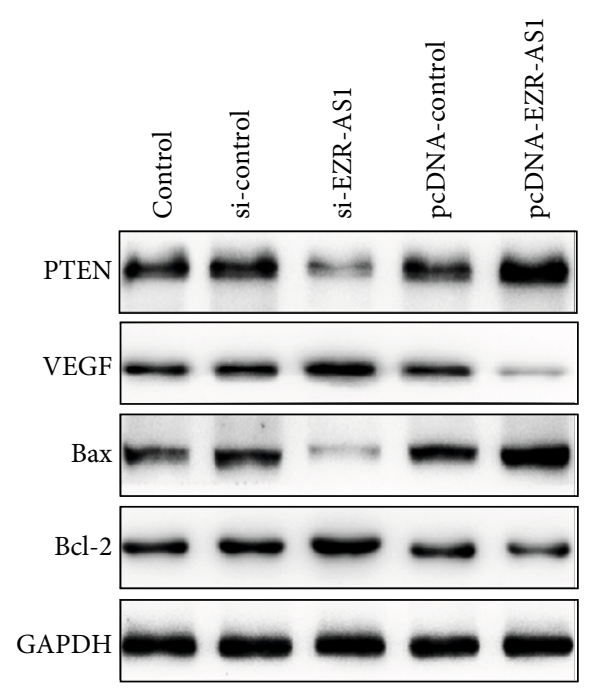

(a)

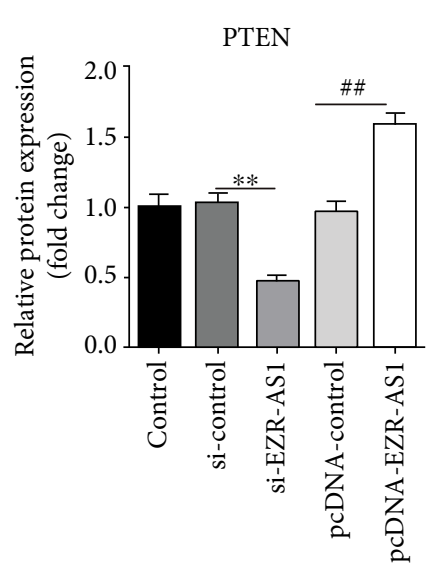

(b)

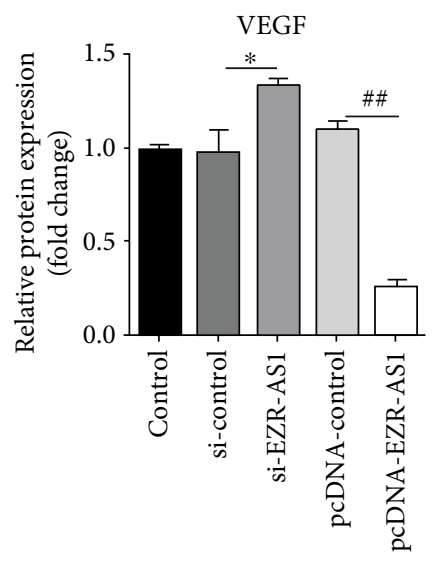

(c)

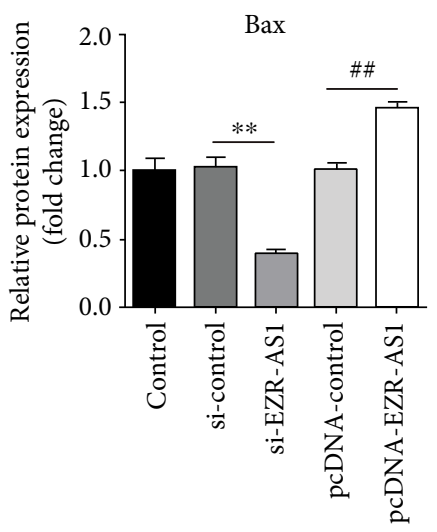

(d)

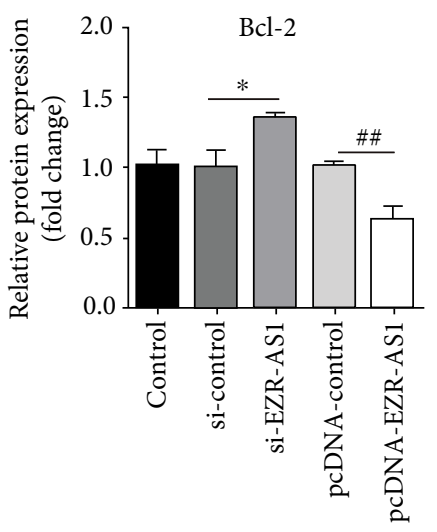

(e)

FIGURE 3: Effects of lncRNA EZR-AS1 on apoptosis-related protein levels in human venous endothelial cells. (a) Western blotting was used $48 \mathrm{~h}$ after transfection to detect the protein expression levels of (b) PTEN, (c) VEGF, (d) Bax, and (e) Bcl-2. All results were expressed as mean \pm SD. ${ }^{*} P<0.05$ and ${ }^{* *} P<0.01$ vs. si-control; ${ }^{\#} P<0.05$ and ${ }^{\# \#} P<0.01$ vs. pcDNA-control.

2.5. Transwell Assay. The cells $\left(5 \times 10^{4} /\right.$ plate $)$ were resuspended in $0.1 \mathrm{ml}$ serum-free culture medium and seeded into the upper chamber of a Transwell insert ( $8 \mu \mathrm{m}$ pore size, 24 well; Corning Inc.). In the lower chamber, $0.8 \mathrm{ml}$ complete culture medium was added as a chemoattractant. Following incubation at $37^{\circ} \mathrm{C}$ for $48 \mathrm{~h}$, the cells that had migrated to the lower surface were fixed with methanol for $20 \mathrm{~min}$ and then stained with $0.1 \%$ crystal violet solution for a further $20 \mathrm{~min}$. Finally, the stained cells were counted under an inverted microscope (Olympus Corporation) at $\times 100$ magnification.

2.6. Flow Cytometry. HUVECs were cultured in 6-well plates to $70-90 \%$ confluence. The cells were then digested using trypsin and collected by centrifugation $\left(1000 \mathrm{rpm}\right.$ at $4^{\circ} \mathrm{C}$ for $5 \mathrm{~min}$ ). Following two washes with precooled PBS, the cells were collected and resuspended in $500 \mu \mathrm{l}$ binding buffer (1X); $5 \mu \mathrm{l}$ Annexin V-APC and $5 \mu \mathrm{l}$ 7-AAD (Nanjing KeyGen Biotech Co., Ltd.) were added, and the cells were incubated at room temperature in the dark for $15 \mathrm{~min}$. Apoptotic cells were then analyzed using a FACScan flow cytometer (BD Biosciences).

2.7. Reverse Transcription-Quantitative PCR (RT-qPCR). The extraction of RNA from the peripheral blood samples was conducted using Total RNA Extraction Kit of blood according to the manufacturer's instructions (BioTeke Corporation). Total RNA was isolated from cells using TRIzol ${ }^{\circledR}$ reagent (Invitrogen; Thermo Fisher Scientific, Inc.). The RNA was then reverse transcribed into first-strand cDNA using the PrimeScript RT Reagent Kit (Takara Bio, Inc.), and the expression levels of the target RNA were determined 

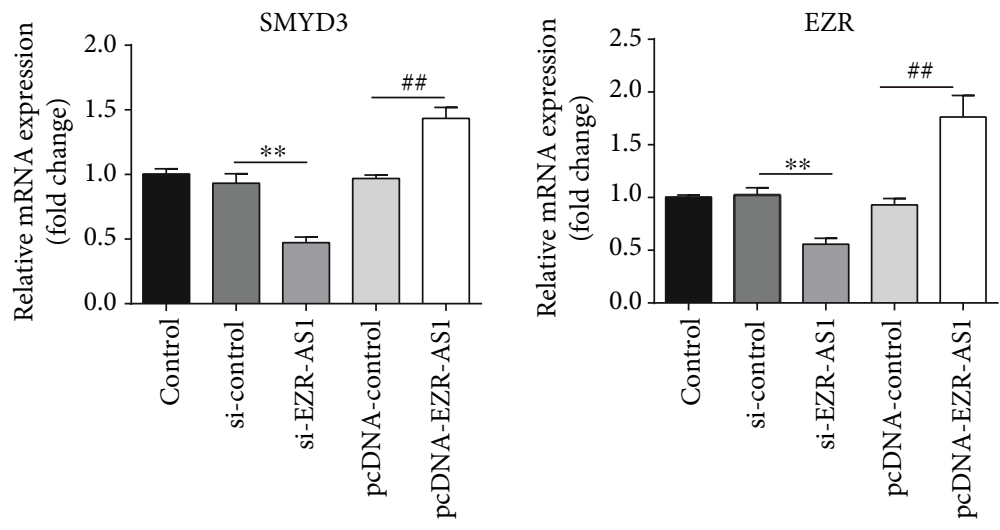

(a)
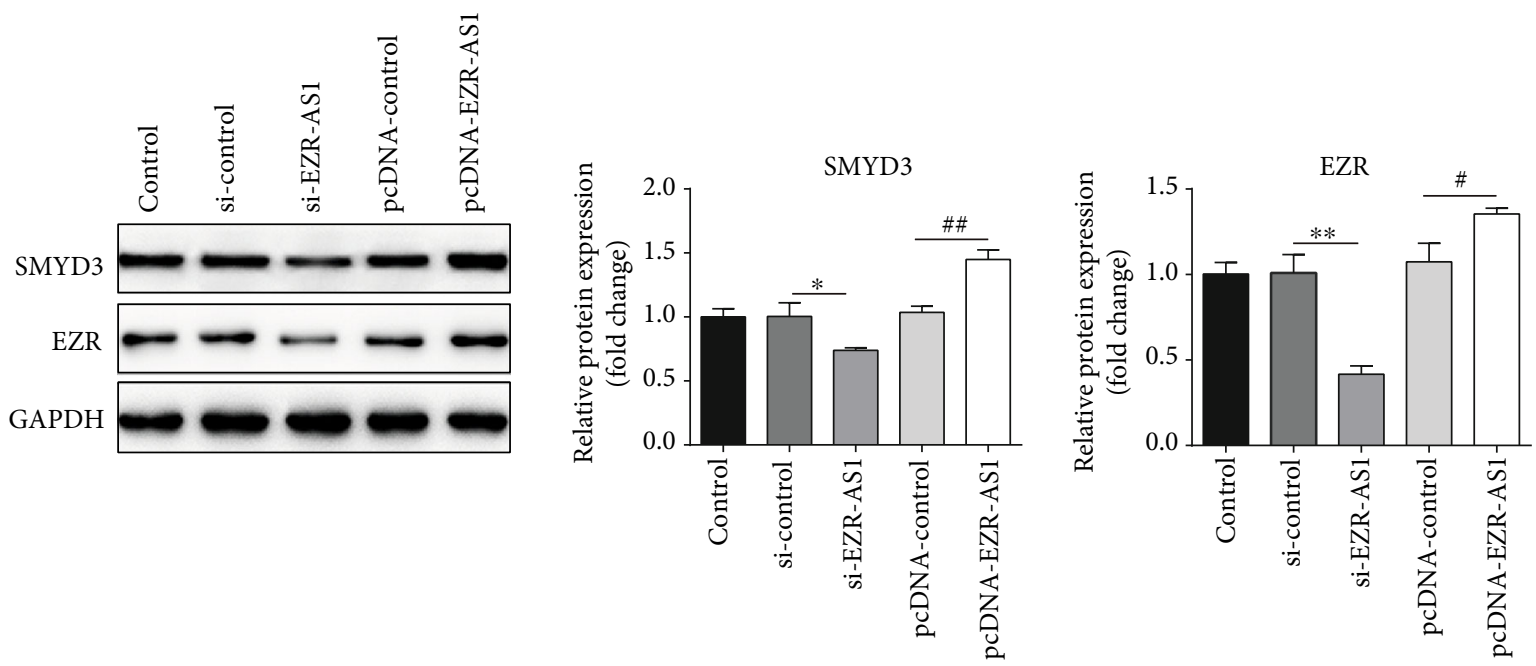

(b)

Figure 4: SMYD3 and EZR are positively regulated by lncRNA EZR-AS1. The (a) mRNA and (b) protein expression levels of SMYD3 and EZR were detected in cells transfected with si-EZR-AS1 or pcDNA-EZR-AS1. All results were expressed as mean \pm SD. ${ }^{*} P<0.05$ and ${ }^{* *} P<0.01$ vs. si-control; ${ }^{\#} P<0.05$ and ${ }^{\# \#} P<0.01$ vs. pcDNA-control.

by RT-qPCR analysis using the Two-Step SYBR PrimeScript RT-PCR Kit (Takara Bio, Inc.) on an Illumina P05775 system (Illumina, Inc.). Glyceraldehyde 3-phosphate dehydrogenase (GAPDH) was used as the normalization control, and the thermocycling conditions were as follows: one cycle at $95^{\circ} \mathrm{C}$ for $1 \mathrm{~min} ; 40$ cycles at $95^{\circ} \mathrm{C}$ for $15 \mathrm{sec}$ and $60^{\circ} \mathrm{C}$ for $1 \mathrm{~min}$; and one cycle at $95^{\circ} \mathrm{C}$ for $15 \mathrm{sec}, 60^{\circ} \mathrm{C}$ for $1 \mathrm{~min}$, and $95^{\circ} \mathrm{C}$ for $15 \mathrm{sec}$. The primers were supplied by Sangon Biotech Co., Ltd., and the sequences are as follows: lncRNA EZRAS1, forward 5'-CCAATGAAGCCTCTCCCGTC-3' and reverse $5^{\prime}$-GGGAGATAACAGGCCCTGAC-3'; EZR, forward $5^{\prime}$-GTGTGGTACTTTGGCCTCCA-3' and reverse $5^{\prime}$-AACTTGGCCCGGAACTTGAA-3'; SMYD3, forward $5^{\prime}$-CCCTCGGGCGTACGTG- $3^{\prime}$ and reverse $5^{\prime}$-CTTGGC GGTTGCGAACTTTT-3' ; and GAPDH, forward $5^{\prime}$-AGC CACATCGCTCAGACAC- $3^{\prime}$ and reverse $5^{\prime}$-GCCCAATA CGACCAAATCC- $3^{\prime}$. Relative gene expression was calculated using the $2^{-\Delta \Delta \mathrm{Cq}}$ method [18].

2.8. Western Blotting. HUVECs were seeded into 6-well cell culture plates and incubated at $37^{\circ} \mathrm{C}$ overnight. The following day, the cells were transfected with the corresponding siRNAs or overexpression pcDNA. Radioimmunoprecipitation assay buffer (Beijing Solarbio Science \& Technology Co., Ltd.) supplemented with complete protease inhibitor cocktail (Roche Molecular Diagnostics) was used to lyse the cells, and the protein concentration was quantified using a bicinchoninic acid protein assay kit (Beyotime Institute of Biotechnology). $40 \mu \mathrm{g}$ of protein extract was separated by SDS-PAGE (using 10 or $12 \%$ gels) and transferred to polyvinylidene fluoride membranes (EMD Millipore). The membranes were blocked for $2 \mathrm{~h}$ with $5 \%$ nonfat milk (at room temperature) and then incubated with primary antibodies at $4^{\circ} \mathrm{C}$ overnight. The membranes were subsequently washed with TBST $(0.1 \%$ Tween 20) and then incubated with the secondary antibody (cat. no. bs-40296G-HRP; $1: 5,000$; BIOSS) for $1.5 \mathrm{~h}$ at room temperature. An enhanced chemiluminescence kit (EMD Millipore) was used to visualize the blots, and the protein bands were quantified using ImageJ 180 software (National Institutes of Health). The primary antibodies used in the present study were as follows: anti-SMYD3 (cat. no. 12859; $1: 1,000$ ), anti-EZR (cat. no. 3145; 1:1,000), antiphosphatase and tensin homolog (PTEN; cat. no. 9188; 


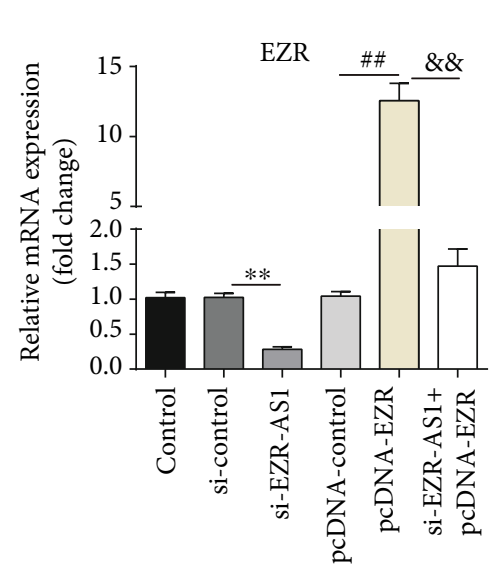

(a)

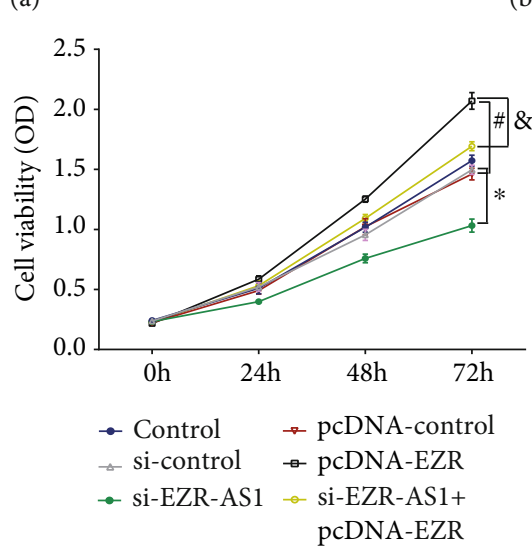

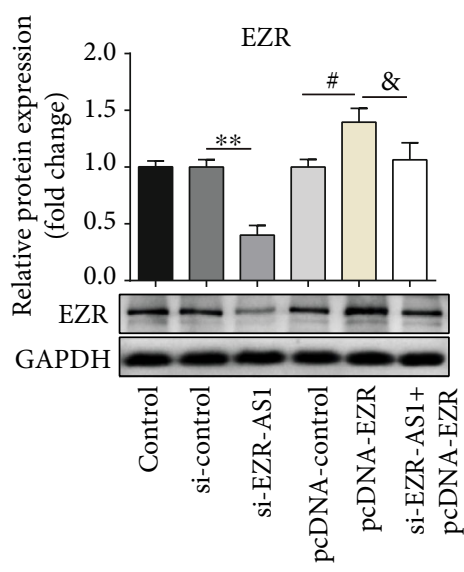

(b)

(c)

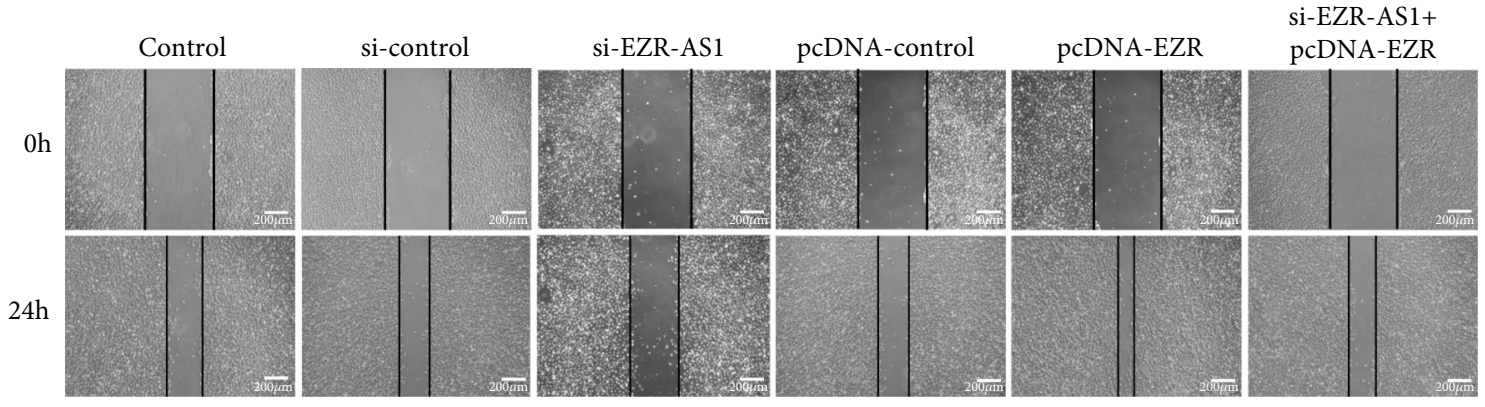

(d)
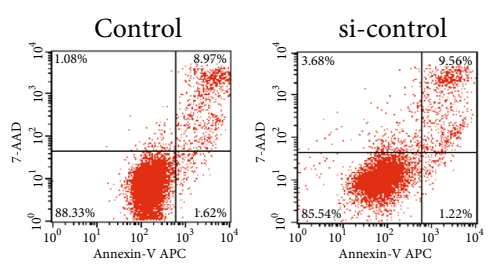

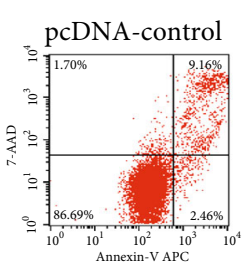

(e)
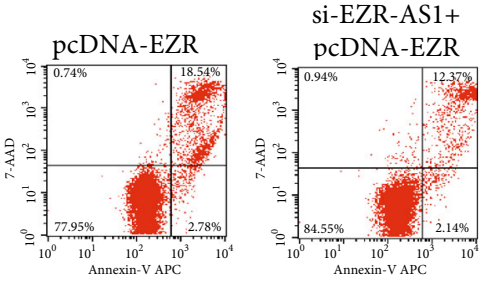

Figure 5: Continued. 


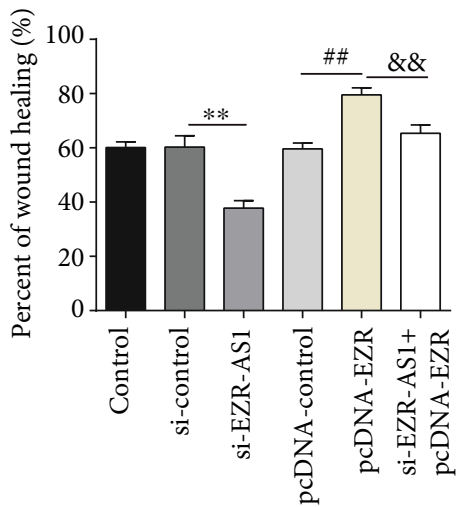

(f)

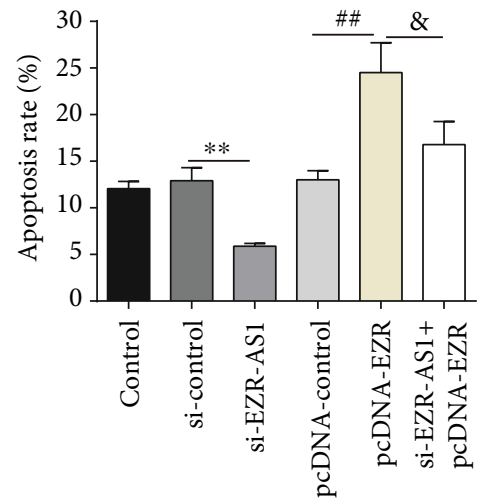

$(\mathrm{g})$

FIGURE 5: Effects of pcDNA-EZR on human venous endothelial cells. After transfected with pcDNA-EZR or si-EZR-AS1+pcDNA-EZR, the (a) mRNA and (b) protein expression levels of EZR were determined using reverse transcription-quantitative PCR and Western blotting. The (c) viability, (d, f) migratory ability, and (e, g) apoptosis of cells were detected using the CCK-8 assay, wound healing assay, and flow cytometry, respectively. Wound healing was quantified. Magnification, $\times 100$ (migration). All results were expressed as mean \pm SD. ${ }^{*} P<0.05$ and ${ }^{* *} P<0.01$ vs. si-control. ${ }^{\#} P<0.05$ and ${ }^{\# \#} P<0.01$ vs. pcDNA-control. ${ }^{\&} P<0.05$ and ${ }^{\& \&} P<0.01$ vs. pcDNA-EZR.

$1: 1,000)$, anti-Bcl-2-associated X protein (Bax; cat. no. 5023; $1: 1,000)$, anti-B cell lymphoma/lewkmia-2 (Bcl-2; cat. no. 4223; $1: 1,000$ ) (all Cell Signaling Technology, Inc.), antivascular endothelial growth factor (VEGF; cat. no. Sc-7269; 1 : 1,000; Santa Cruz Biotechnology), and anti-GAPDH (cat. no. AB-P-R001; $1: 1,000$; Goodhere, Inc.).

2.9. Statistical Analysis. Each experiment was repeated $\geq 3$ times, and numerical data are presented as mean \pm SD. The differences between the means were analyzed using Student's unpaired $t$-tests or one-way ANOVA where appropriate. All statistical analyses were performed using GraphPad 7.0 software (GraphPad Software, Inc.), and $P<0.05$ was considered to indicate a statistically significant difference.

\section{Results}

3.1. Expression of $\operatorname{lncRNA} E Z R-A S 1$ and EZR in the Peripheral Blood. Initially, RT-qPCR was used to analyze the expression of EZR-AS1 and EZR in the peripheral blood of 35 patients with $\mathrm{CHD}$ and 38 control subjects. The results revealed that EZR-AS1 expression was markedly upregulated in patients with $\mathrm{CHD}$, compared with the controls (Figure 1(a)). Similarly, EZR expression was also elevated in patients with CHD (Figure 1(b)).

3.2. Effects of IncRNA EZR-AS1 on Proliferation, Migration, and Apoptosis in HUVECs. To explore the functions of EZR-AS1 in HUVECs, the cells were transfected with siRNA or overexpression pcDNA of EZR-AS1; transfection efficiency is illustrated in Figure 2(a). The results of the CCK-8 assay showed that $E Z R-A S 1$ knockdown reduced cell viability (Figure 2(b)). Additionally, si-EZR-AS1 suppressed the migration (Figures 2(c) and 2(d)) and apoptosis (Figure 2(e)) of HUVECs. Overexpression of EZR-AS1 resulted in the reverse effect.

3.3. Effects of IncRNA EZR-AS1 on the Expression Levels of Apoptosis-Related Proteins in HUVECs. To determine whether lncRNA EZR-AS1 mediated apoptosis, Western blotting was used to detect the expression levels of apoptosis-related proteins, including PTEN, VEGF, Bax, and Bcl-2. EZR-AS1 knockdown increased the expression levels of VEGF and Bcl-2, while decreasing those of PTEN and Bax. The opposite effect was observed when EZR-AS1 was overexpressed (Figures 3(a)-3(e)). These data indicate that downregulating the expression of IncRNA EZR-AS1 inhibits apoptosis in HUVECs.

3.4. IncRNA EZR-AS1 Positively Regulates SMYD3 and EZR Expression. The expression levels of SMYD3 and EZR were analyzed via lncRNA EZR-AS1 knockdown or overexpression. The results showed that the downregulation of EZRAS1 decreased the expression of SMYD3 and EZR, and the opposite effect was observed when EZR-AS1 was overexpressed. These results indicate that lncRNA EZR-AS1 positively regulates the expression of SMYD3 and EZR (Figures 4(a) and 4(b)).

3.5. Overexpression of EZR Reverses the Effects of EZR-AS1 Knockdown on the Biological Properties of HUVECs. To further explore whether EZR was involved in the effects of siEZR-AS1, HUVECs were transfected with an EZR overexpression pcDNA. RT-qPCR and Western blotting confirmed that the EZR expression level was significantly decreased in cells transfected with both si-EZR-AS1 and pcDNA-EZR (Figures 5(a) and 5(b)), compared with those transfected with pcDNA-EZR alone. Proliferation, migration, and apoptotic capacity were evaluated after transfection; the results showed that the overexpression of EZR significantly reversed the effects of si-EZR-AS1 on cell proliferation, migration, and apoptosis (Figures 5(c)-5(g)).

3.6. Overexpression of SMYD3 Reverses the Effect of si-EZRAS1 on the Biological Properties of HUVECs. Finally, to investigate the involvement of SMYD3 in the effects of lncRNA EZR-AS1, HUVECs were transfected with pcDNA-SMYD3 


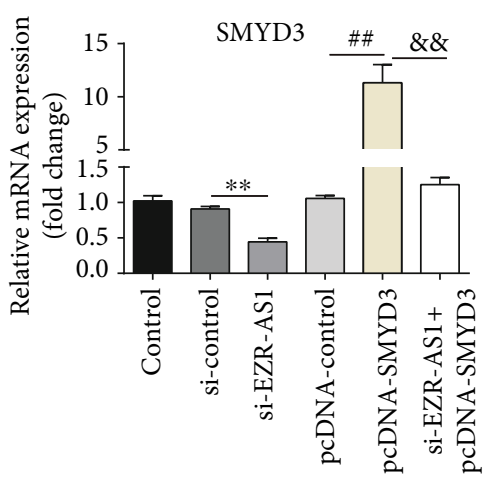

(a)

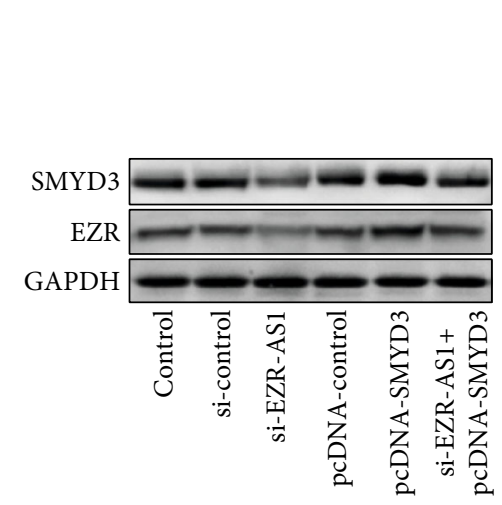

(c) (b)

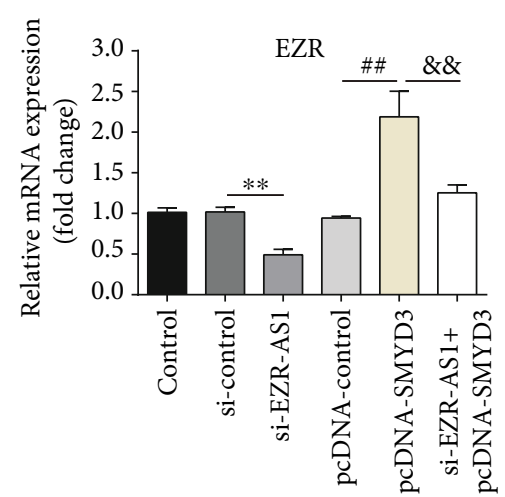

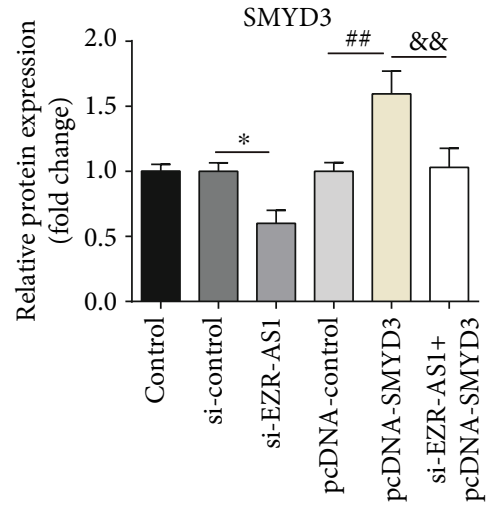

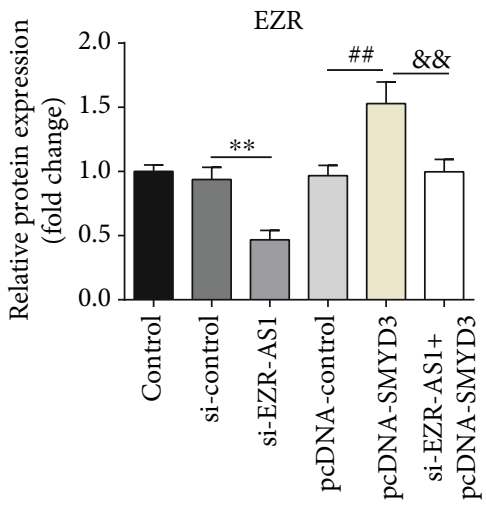

(d)

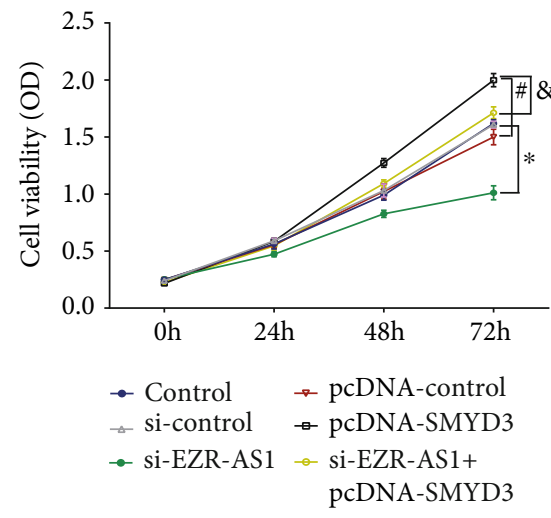

(e)

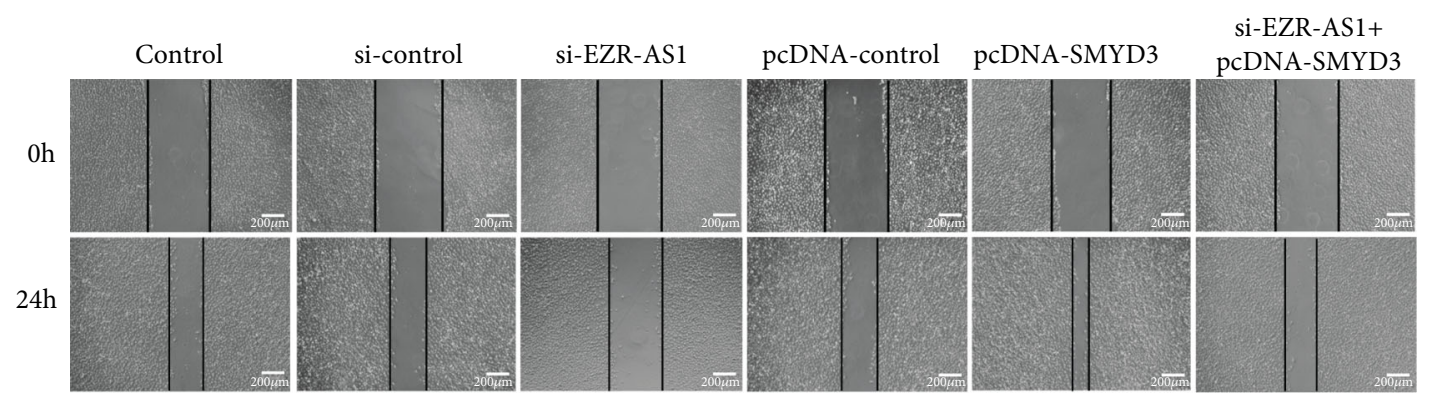

(f)

FIgURE 6: Continued. 

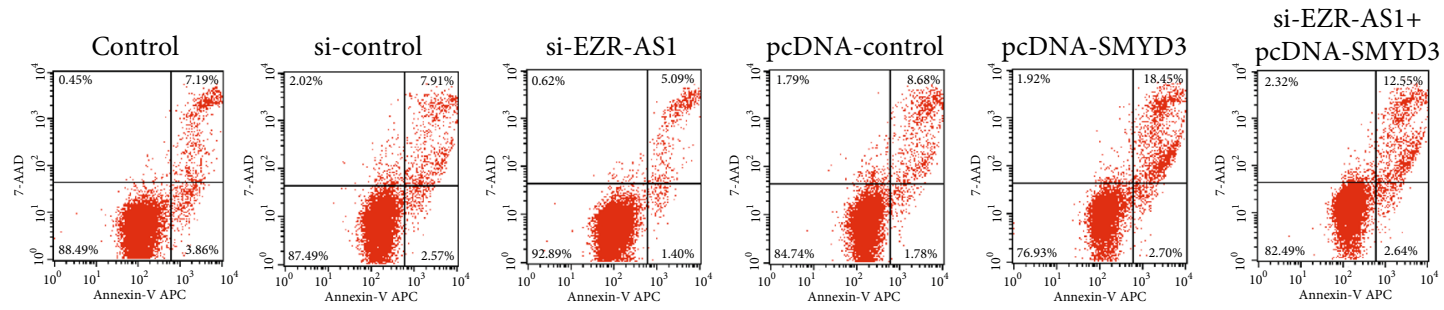

(g)

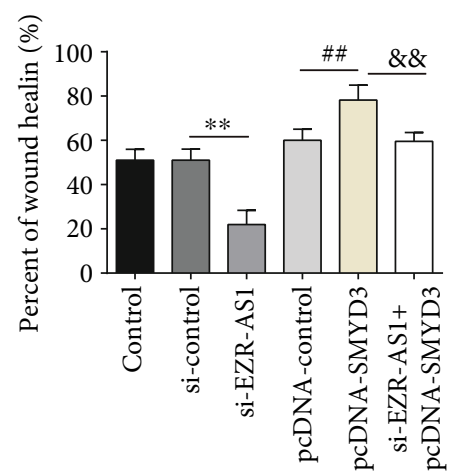

(h)

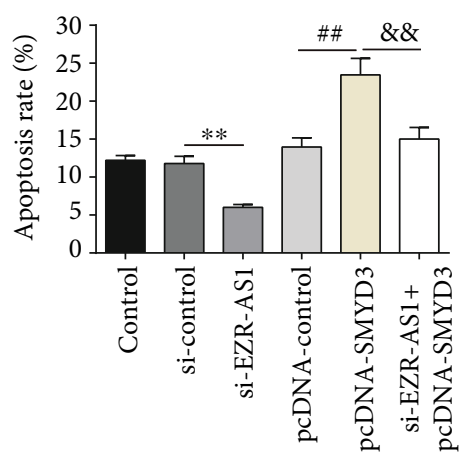

(i)

FIGURE 6: EZR-AS1 mediates EZR expression via SMYD3. After transfected with pcDNA-SMYD3 or si-EZR-AS1+pcDNA-SMYD3, the (a, b) mRNA and $(c, d)$ protein expression levels of SMYD3 and EZR were determined by reverse transcription-quantitative PCR and Western blotting. (e) Cell viability was detected by CCK-8 assay. (f, h) Migratory ability was detected by wound healing assay. (g, i) Apoptosis was detected by flow cytometry. Wound healing was quantified. Magnification, $\times 100$ (migration). All results were expressed as mean \pm SD. ${ }^{*} P$ $<0.05$ and ${ }^{* *} P<0.01$ vs. si-control. ${ }^{\#} P<0.05$ and ${ }^{\# \#} P<0.01$ vs. pcDNA-control. ${ }^{\circledR} P<0.05$ and ${ }^{\& \&} P<0.01$ vs. pcDNA-SMYD3.

or si-EZR-AS1+pcDNA-SMYD3. The results indicated that SMYD3 overexpression could reverse the effects of EZRAS1 knockdown, resulting in the upregulation of SMYD3 and EZR (Figures 6(a)-6(d)). At the same time, the upregulation of SMYD3 reversed the proliferation, migration, and apoptotic ability of si-EZR-AS1-transfected cells (Figures 6(e)-6(i)).

\section{Discussion}

Cardiovascular diseases, particularly CHD and stroke, remain the leading global causes of death, and the lifetime risk of CHD is $67 \%$ in individuals $>55$ years of age [19-21]. Therefore, new strategies for the treatment of $\mathrm{CHD}$ are urgently required. lncRNAs have been associated with the treatment of various cardiovascular diseases, including CHD [22, 23]; thus, defining the functions of lncRNAs may aid in the development of novel diagnostic and therapeutic targets for CHD.

Generally speaking, CHD is diagnosed when stenosis of the coronary artery reaches $>50 \%$. In the study, RT-qPCR was used to analyze the expression of EZR-AS1 in the peripheral blood of patients with severe CHD (with stenosis $>80 \%$ ) and controls. The results showed that EZR-AS1 was robustly upregulated in patients with severe CHD. To the best of our knowledge, this was the first study to have evaluated the role of lncRNA EZR-AS1 in CHD. Based on these findings, the functions of lncRNA EZR-AS1 were further assessed through in vitro experimentation.
Vascular endothelial cells are important components of the vascular wall. The abnormal proliferation and migration of vascular endothelial cells are important pathophysiological processes in diseases such as CHD, hypertension, and restenosis after percutaneous coronary intervention [24-26]. In the present study, the role of EZR-AS1 was evaluated in HUVECs by assessing cell proliferation, migration, and apoptosis; this was achieved by transfection with si-EZR-AS1 or an overexpression pcDNA. The results revealed that the downregulation of EZR-AS1 significantly inhibited HUVECs proliferation, migration, and apoptosis; the opposite effects were observed following EZR-AS1 overexpression. These findings suggest that lncRNA EZR-AS1 influences the malignant behaviors of HUVECs, providing a basis for its therapeutic application in CHD.

EZR, encoded by Vil2, was identified as the first member of the ERM family. EZR is upregulated in a number of diseases, such as breast and cervical cancer, and overexpression of the EZR gene may enhance the metastatic capacity of tumors [27, 28]. Although the function of EZR has been extensively studied, the transcriptional regulation of the EZR gene is still poorly understood. To the best of our knowledge, the role of EZR in the progression of $\mathrm{CHD}$ remains unclear. In the current study, EZR was revealed to be upregulated in patients with $\mathrm{CHD}$, and as predicted, increased levels of EZR promoted the proliferation and migration of HUVECs. Similar to the effects of the lncRNAs BDNF-AS and NEXN-AS1 on target gene expression [29, 30], knocking down EZR-AS1 decreased the expression level of EZR. Additionally, EZR overexpression reversed the effects of EZR-AS1 
knockdown, indicating that EZR is involved in regulating the biological behaviors of HUVECs. Furthermore, siRNA suppressed the expression of EZR-AS1 while simultaneously raised that of SMYD3, which could reverse the downregulation of EZR mediated by the si-EZR-AS1, which suggested that $E Z R-A S 1$ is most likely to regulate EZR expression through SMYD3 signaling in HUVECs; more experiments such as RNA-binding protein immunoprecipitation (RIP) and chromatin immunoprecipitation (ChIP) should be done in our next work to further confirm it.

\section{Conclusion}

In conclusion, and to the best of our knowledge, the present study is the first to report that lncRNA EZR-AS1 is upregulated in severe CHD and that the downregulation of EZRAS1 inhibits the proliferation, migration, and apoptosis of HUVECs via SMYD3. These data facilitate a deeper understanding of the molecular mechanisms of lncRNA EZR-AS1 in CHD. Therefore, lncRNA EZR-AS1 may be a potential biomarker for the diagnosis and treatment of severe CHD.

\section{Data Availability}

The analyzed data sets used and/or analysed during the present study are available from the corresponding author on reasonable request.

\section{Conflicts of Interest}

The authors declare that they have no conflicts of interests.

\section{Authors' Contributions}

QW designed the research. GY conducted the experimental protocols with assistance from XL, FS, and JH. GY, MT, YX, and SD analyzed the data and wrote the manuscript. All authors reviewed the results and approved the final manuscript.

\section{Acknowledgments}

The present study was supported by the Science Technology Platform and Talent Team Plan Projects in Guizhou Province (grant no. 20175405) and the High-level Innovation Talents Training Program in Guizhou Province (grant no. 20164023).

\section{References}

[1] GBD 2016 Causes of Death Collaborators, "Global, regional, and national age-sex specific mortality for 264 causes of death, 1980-2016: a systematic analysis for the Global Burden of Disease Study 2016," The Lancet, vol. 390, no. 10100, pp. 11511210, 2016.

[2] GBD 2016 Mortality Collaborators, "Global, regional, and national age-sex specific mortality for 264 causes of death, 1980-2016: a systematic analysis for the Global Burden of Disease Study 2016," The Lancet, vol. 390, no. 10100, pp. 11511210, 2017.
[3] J. J. DiNicolantonio, S. C. Lucan, and J. H. O'Keefe, "The evidence for saturated fat and for sugar related to coronary heart disease," Progress in Cardiovascular Diseases, vol. 58, no. 5, pp. 464-472, 2016.

[4] J. R. Lewis, S. Radavelli-Bagatini, L. Rejnmark et al., "The effects of calcium supplementation on verified coronary heart disease hospitalization and death in postmenopausal women: a collaborative meta-analysis of randomized controlled trials," Journal of Bone and Mineral Research, vol. 30, no. 1, pp. 165175, 2015.

[5] I. Martianov, A. Ramadass, A. Serra Barros, N. Chow, and A. Akoulitchev, "Repression of the human dihydrofolate reductase gene by a non-coding interfering transcript," Nature, vol. 445, no. 7128, pp. 666-670, 2007.

[6] J. J. Quinn and H. Y. Chang, "Unique features of long noncoding RNA biogenesis and function," Nature Reviews Genetics, vol. 17, no. 1, pp. 47-62, 2016.

[7] M. Boulberdaa, E. Scott, M. Ballantyne et al., "A role for the long noncoding RNA SENCR in commitment and function of endothelial cells," Molecular Therapy, vol. 24, no. 5, pp. 978-990, 2016.

[8] S. Greco, M. Gorospe, and F. Martelli, "Noncoding RNA in age-related cardiovascular diseases," Journal of Molecular and Cellular Cardiology, vol. 83, pp. 142-155, 2015.

[9] C. Cai, H. Zhu, X. Ning et al., "LncRNA ENST00000602558.1 regulates ABCG1 expression and cholesterol efflux from vascular smooth muscle cells through a p65-dependent pathway," Atherosclerosis, vol. 285, pp. 31-39, 2019.

[10] Q. Lu, Q. Meng, M. Qi, F. Li, and B. Liu, "Shear-sensitive lncRNA AF131217.1 inhibits inflammation in HUVECs via regulation of KLF4," Hypertension, vol. 73, no. 5, pp. e25e34, 2019.

[11] M. Ponnusamy, F. Liu, Y. Zhang et al., "Long noncoding RNA $\mathrm{CPR}$ (cardiomyocyte proliferation regulator) regulates cardiomyocyte proliferation and cardiac repair," Circulation, vol. 139, no. 23, pp. 2668-2684, 2019.

[12] M. Arpin, D. Chirivino, A. Naba, and I. Zwaenepoel, "Emerging role for ERM proteins in cell adhesion and migration," Cell Adhesion \& Migration, vol. 5, no. 2, pp. 199-206, 2014.

[13] H. Cho, G. Q. Shen, X. Wang et al., "Long noncoding RNAANRILregulates endothelial cell activities associated with coronary artery disease by up-regulatingCLIP1,EZR, andLYVE1genes," The Journal of Biological Chemistry, vol. 294, no. 11, pp. 3881-3898, 2019.

[14] M. E. Sarris, P. Moulos, A. Haroniti, A. Giakountis, and I. Talianidis, "Smyd3 is a transcriptional potentiator of multiple cancer-promoting genes and required for liver and colon cancer development," Cancer Cell, vol. 29, no. 3, pp. 354366, 2016.

[15] Y. Wang, B. Xie, W. Lin et al., “Amplification of SMYD3 promotes tumorigenicity and intrahepatic metastasis of hepatocellular carcinoma via upregulation of CDK2 and MMP2," Oncogene, vol. 38, no. 25, pp. 4948-4961, 2019.

[16] X.-D. Zhang, G. W. Huang, Y. H. Xie et al., "The interaction of lncRNA EZR-AS1 with SMYD3 maintains overexpression of EZR in ESCC cells," Nucleic Acids Research, vol. 46, no. 4, pp. 1793-1809, 2018.

[17] Y. Bai, X. Zhou, L. Huang, Y. Wan, X. Li, and Y. Wang, "Long noncoding RNA EZR-AS1 promotes tumor growth and metastasis by modulating $\mathrm{Wnt} / \beta$-catenin pathway in breast 
cancer," Experimental and Therapeutic Medicine, vol. 16, no. 3, pp. 2235-2242, 2018.

[18] K. J. Livak and T. D. Schmittgen, "Analysis of relative gene expression data using real-time quantitative PCR and the $2^{-\Delta \Delta C_{\mathrm{T}}}$ method," Methods, vol. 25, no. 4, pp. 402-408, 2001.

[19] The Global Burden of Metabolic Risk Factors for Chronic Diseases Collaboration, "Metabolic mediators of the effects of body-mass index, overweight, and obesity on coronary heart disease and stroke: a pooled analysis of 97 prospective cohorts with 1.8 million participants," The Lancet, vol. 383, no. 9921, pp. 970-983, 2014.

[20] L. Yang, Y. Liu, S. Wang, T. Liu, and H. Cong, “Association between Lp-PLA2 and coronary heart disease in Chinese patients," Journal of International Medical Research, vol. 45, no. 1, pp. 159-169, 2017.

[21] C. Berry, D. Corcoran, B. Hennigan, S. Watkins, J. Layland, and K. G. Oldroyd, "Fractional flow reserve-guided management in stable coronary disease and acute myocardial infarction: recent developments," European Heart Journal, vol. 36, no. 45, pp. 3155-3164, 2015.

[22] G. Wu, J. Cai, Y. Han et al., "LincRNA-p21 regulates neointima formation, vascular smooth muscle cell proliferation, apoptosis, and atherosclerosis by enhancing p53 activity," Circulation, vol. 130, no. 17, pp. 1452-1465, 2014.

[23] B. Yan, J. Yao, J. Y. Liu et al., "IncRNA-MIAT regulates microvascular dysfunction by functioning as a competing endogenous RNA," Circulation Research, vol. 116, no. 7, pp. 11431156, 2015.

[24] Y. Li, J. Huang, Z. Jiang, Y. Jiao, and H. Wang, "FGF21 inhibitor suppresses the proliferation and migration of human umbilical vein endothelial cells through the eNOS/PI3K/AKT pathway," American Journal of Translational Research., vol. 9, no. 12, pp. 5299-5307, 2017.

[25] Q.-H. Wu, Y. Ma, C. C. Ruan et al., "Loss of osteoglycin promotes angiogenesis in limb ischaemia mouse models via modulation of vascular endothelial growth factor and vascular endothelial growth factor receptor 2 signalling pathway," Cardiovascular Research, vol. 113, no. 1, pp. 70-80, 2017.

[26] B. Hao, Y. Xiao, F. Song et al., "Metformin-induced activation of AMPK inhibits the proliferation and migration of human aortic smooth muscle cells through upregulation of p53 and IFI16," International Journal of Molecular Medicine, vol. 41, no. 3, pp. 1365-1376, 2018.

[27] J. Jeong, J. Choi, W. Kim et al., "Inhibition of ezrin causes PKC $\alpha$-mediated internalization of erbb 2/ HER2 tyrosine kinase in breast cancer cells," Journal of Biological Chemistry, vol. 294, no. 3, pp. 887-901, 2019.

[28] R. Qin, L. Cao, J. Wang, and J. Liu, "Promoter methylation of ezrin and its impact on the incidence and prognosis of cervical cancer," Cellular Physiology and Biochemistry, vol. 50, no. 1, pp. 277-287, 2018.

[29] F. Modarresi, M. A. Faghihi, M. A. Lopez-Toledano et al., "Inhibition of natural antisense transcripts in vivo results in gene- specific transcriptional upregulation," Nature Biotechnology, vol. 30, no. 5, pp. 453-459, 2012.

[30] Y.-W. Hu, F. X. Guo, Y. J. Xu et al., "Long noncoding RNA NEXN-AS1 mitigates atherosclerosis by regulating the actinbinding protein NEXN," Journal of Clinical Investigation, vol. 129, no. 3, pp. 1115-1128, 2019. 